\section{Avaliação das razões de sucesso na psicoterapia cognitiva}

\section{Sr. Editor,}

Em recente artigo dessa revista, ${ }^{1}$ Cordioli et al avaliaram a eficácia da terapia cognitivo comportamental em grupo (TCCG) para pacientes com transtorno obsessivo compulsivo. A TCCG tem se mostrado efetiva em uma série de problemas psiquiátricos. Os serviços públicos e instituições médicas têm encontrado na terapia cognitiva e, mais ainda, na terapia cognitiva em grupo, uma solução eficaz e prática para lidar com as crescentes demandas de tratamento. A demonstração repetida da eficácia dessa modalidade de terapia é uma importante referência para aqueles que planejam estruturar serviços públicos de psicoterapia.

Como é de se esperar, a efetividade da terapia não poderia ser homogênea. Em todo estudo de tratamentos, alguns clientes melhoram, alguns não, e outros pioram. No estudo em questão, 21,9\% dos pacientes mantiveram sintomas obsessivos significativos no fim do tratamento. Diversas possíveis explicações para o insucesso são citadas pelos autores.

Entendemos que a simples listagem de todos os possíveis fatores envolvidos nessa variação poderia ser longa e desnecessária, e que vários desses fatores, inevitavelmente, deixam de ser citados. Entretanto, consideramos necessário ressaltar o papel significativo da aliança terapêutica como um aspecto importante na efetividade de todo e qualquer processo terapêutico.

Diversas evidências têm surgido nas últimas décadas correlacionando diretamente a aliança terapêutica com a efetividade da psicoterapia: quanto maior ou de mais qualidade a aliança terapêutica, maior a efetividade da terapia. Morgan et $\mathrm{al}^{2}$ encontraram uma correlação significativa entre a aliança terapêutica e o sucesso da terapia. Strupp ${ }^{3}$ notou que o resultado da terapia é significativamente determinado pelo quanto o paciente aproveita da interação particular que o terapeuta pode oferecer. Considerou também igualmente importantes para os resultados tanto a habilidade do paciente em se envolver numa relação terapêutica quanto a resolução das rupturas da aliança terapêutica. Luborsky et al, ${ }^{4}$ por sua vez, também consideraram que a aliança terapêutica foi o fator comum entre diversas modalidades de psicoterapia, fator esse significativamente responsável pela eficácia da terapia.

A aliança terapêutica tem sido considerada um elemento fundamental em todas as formas de psicoterapia. ${ }^{5}$ Um número crescente de autores na tradição cognitivo-comportamental tem enfatizado a importância de considerar o relacionamento terapêutico como instrumento de mudança. ${ }^{5}$ Dada a importância da aliança terapêutica, é essencial sua consideração na análise das razões de sucesso ou não de um projeto de pesquisa em psicoterapia. A omissão desse fator pode obscurecer as razões de insucesso de diversas situações terapêuticas, apesar da aplicação correta e adequada das técnicas em questão.

\author{
Carlos Eduardo Reche \\ Universidade do Estado de Minas Gerais \\ Núcleo Mineiro de Terapias Cognitivas \\ Henrique Alvarenga da Silva \\ Universidade Federal São João Del-Rei \\ Núcleo Mineiro de Terapias Cognitivas
}

\section{Referências}

1. Cordioli AV, Heldt E, Bochi DB, Margis R, Sousa MB, Tonello JF et al. Cognitive-behavioral group therapy in obsessive-compulsive disorder: a clinical trial. Rev Bras Psiq 2002;24(3):113-20.

2. Morgan R, Luborsky L, Crits-Christoph P, Curtis H, Solomon J. Predicting the outcomes of psychotherapy by the Penn Helping Alliance Rating Method. Arch Gen Psychiatry 1982;39(4):397-402.

3. Strupp HH. Sucess and failure of time limited psychotherapy. Further evidence. Comparision IV. Arch Gen Psychiatry 1980;37:947-54.

4. Luborsky L, Singer B, Luborsky L. Comparative studies of psychotherapies: is it true that "Everyone has won and must have all prizes?" Arch Gen Psychiatry 1975;32:995-1008.

5. Safran JD. Ampliando os limites da terapia cognitiva: o relacionamento terapêutico, a emoção e o processo de mudança. Porto Alegre: Artmed; 2002.

\section{Aristides Volpato Cordioli responde:}

\section{Sr. Editor,}

Resumidamente, os resultados de uma psicoterapia dependem:

1) da natureza do transtorno de que o paciente é portador;

2) da(s) técnica(s) utilizada(s);

3) das condições pessoais do paciente para cumprir as exigências da técnica e vincular-se ao terapeuta;

4) da pessoa do terapeuta: sua competência, seu estilo, de qualidades pessoais como empatia, autenticidade, entusiasmo, calor humano, capacidade de inspirar confiança, de estabelecer um vínculo com o paciente; e finalmente

5) da qualidade da relação paciente-terapeuta: o vínculo e a capacidade do par de levar adiante as tarefas psicoterápicas - a Aliança Terapêutica (AT).

O transtorno obsessivo-compulsivo (TOC) é um transtorno heterogêneo, com sua etiopatogenia ainda pouco esclarecida, com distintas apresentações clínicas e respostas aos tratamentos. A gravidade dos sintomas, a presença de comorbidades, a intensidade e a fixidez de crenças disfuncionais, e a adesão aos exercícios de Exposição e Prevenção da Resposta (EPR) parecem influenciar os resultados dos tratamentos. ${ }^{1,2}$ É possível que estes fatores, por si só, expliquem a ineficácia da psicoterapia, 
mesmo num contexto interpessoal favorável.

Quanto à técnica, tem sido comprovada a eficácia da terapia comportamental de EPR e da terapia cognitiva no TOC. Ensaios clínicos que utilizaram nos grupos controles psico-educação ou técnicas de controle da ansiedade, não reduziram os sintomas. ${ }^{3} \mathrm{O}$ quanto é do nosso conhecimento, não têm sido investigadas na Terapia Cognitivo-Comportamental do TOC as influências nos resultados dos aspectos pessoais do terapeuta e do paciente e da relação terapêutica, incluindo o vínculo e a Aliança Terapêutica (AT).

AT é um conceito surgido no âmbito da psicanálise e da psicoterapia de orientação analítica e valoriza a capacidade do paciente em seguir as compreensões do analista, tornando-se um colaborador ativo na busca do insight, e, da parte do analista, a capacidade de compreender o paciente e identificar-se com ele., ${ }^{4,5}$ A transposição pura e simples deste conceito para o âmbito da TCC no TOC parece problemática. Nesta modalidade de tratamento são cruciais: a adesão aos exercícios de EPR, a capacidade de tolerar o aumento da ansiedade, de identificar pensamentos automáticos e crenças disfuncionais, a flexibilidade para questioná-las e substituí-las por pensamentos realistas. $\mathrm{O}$ terapeuta deve ser capaz de motivar o paciente a enfrentar medos ao realizar os exercícios, a questionar crenças arraigadas e a abandonar a solução de neutralizar seus medos, aflições e desconfortos mediante a execução de rituais. Num primeiro momento, seria necessário se chegar a um consenso quanto ao conceito de AT no âmbito da TCC, da TCC no TOC em particular, dos fatores que a compõe e dos critérios operacionais para aferila. Num segundo momento, seriam criados e validados instrumentos para mensurá-la, para posteriormente serem utilizados em projetos de pesquisa. Mas sem dúvida, numa psicoterapia na qual se acredita que técnicas específicas são os ingredientes efetivos, seria uma questão instigante avaliar a influência nos resultados dos chamados fatores não-específicos como aspectos pessoais do terapeuta e do paciente, e do tipo de aliança que conseguem estabelecer em função destas características. Esclarecer estas questões poderia nos auxiliar a compreender as razões pelas quais alguns pacientes não melhoram.

\section{Aristides Volpato Cordioli \\ Departamento de Psiquiatria e Medicina Legal da Faculdade de Medicina da Universidade Federal do Rio Grande do Sul}

\section{Referências}

1. Basoglu M, Lax T, Kasviskis Y, Marks IM. Predictors of improvement in obsessive-compulsive disorder. J Anx Dis 1988;2:299-17.

2. Neziroglu FA, Stevens KP, Yaryura-Tobias JA. Overvalued ideas and their impact on treatment outcome. Rev Bras Psiquiatr 1999;21:209-16.

3. Lindsay M, Crino R, Andrews G. Controlled trial of exposure and response prevention in obsessive-compulsive disorder. Brit J Psychiatry 1997;171:135-9.

4. Greenson R. A transferência. In: A técnica e a prática da psicanálise. Rio de Janeiro: Imago; 1981. p. 210-21.

5. Cordioli AV, Calich JC, Fleck MPA. Aliança terapêutica: uma revisão do conceito. In: Eizirik CL, Aguiar R, Schestatsky S, organizadores. Psicoterapia de orientação analítica: teoria e prática. Porto Alegre: Artes Médicas; 1989. p. 226-36.

\section{Terapia cognitivo-comportamental em grupo no transtorno obsessivo-compulsivo: um ensaio clínico}

\section{Sr. Editor,}

A leitura do artigo em questão chama a atenção pela proposta de um trabalho de grande demanda em nosso país. A terapia em grupo é de grande valia por democratizar um tipo de tratamento ainda muito custoso para a população de um país como o Brasil.

Muitos artigos têm sido publicados relatando a melhora produzida pela realização de sessões de Exposição com Prevenção de Respostas (EPR) em pacientes diagnosticados com Transtorno Obsessivo-Compulsivo (TOC). ${ }^{1}$ Um número maior de artigos relata experimentos de EPR individual.Temos também, a disposição, referências de artigos que relatam estudos com sessões de EPR em grupo para pacientes com TOC. ${ }^{2}$

$\mathrm{O}$ trabalho de Cordioli e equipe sugere que a utilização de técnicas cognitivas pode contribuir na melhora dos sintomas de pacientes com TOC. Considerando que neste estudo foram utilizadas EPR e técnicas cognitivas concomitantemente, não é possível afirmar que uma ou outra variável foi responsável pela melhora encontrada. Estudos comparativos entre grupo com utilização de EPR e grupo com utilização de técnicas cognitivas poderiam indicar quais variáveis controlaram a alteração dos comportamentos característicos do indivíduo com TOC.

Um dado que chama a atenção do leitor, é a boa taxa de adesão $(93,76 \%)$ à terapia em grupo neste estudo. Apenas 6,25\% dos pacientes deixaram o estudo. Quais seriam as variáveis que poderiam explicar esta taxa de adesão? Seria a possibilidade de os pacientes estabelecerem novos vínculos? Sabe-se que o grupo funciona como condição facilitadora para o desenvolvimento de novos vínculos. Sabemos que pacientes com TOC possuem um marcado déficit de habilidades sociais que prediz uma baixa resposta ao tratamento. ${ }^{3}$

Talvez, as mesmas variáveis que controlaram a taxa de adesão favoreçam aqueles pacientes diagnosticados com TOC refratário, que não respondem a EPR individual e ao tratamento farmacológico habitual. Será que esta forma de terapia não poderia ser uma abordagem recomendada mais freqüentemente no tratamento de pacientes com TOC? Neste sentido, faz-se necessário o investimento em estudos como o de Cordioli e equipe.

\section{Priscila de J Chacon, Márcia M Motta e Cristina Belloto \\ Projeto Transtornos do Espectro Obsessivo-Compulsivo (PROTOC) Departamento de Psiquiatria Faculdade de Medicina da Universidade de São Paulo}

\section{Referências}

1. Abramowitz JS. Effectiveness of psychological and pharmacological treatments for obsessive-compulsive disorder: a quantitative review. J Consult Clin Psychol 1997;65:44-52.

2. Van Noppen MSW, Pato MT, Marsland R, Rasmussen SA. A time-limited behavioral group for treatment of obsessive-compulsive disorder. J Psychother Pract Res 7:272-280, October 1998.

3. Steketee G, Eisen J, Dyck I, Warshaw M, Rasmussen S. Predictors of course in obsessive-compulsive disorder. Psychiatry Res 1999;89:229-38. 